\title{
MINIMAL HARMONIC FUNCTIONS ON DENJOY DOMAINS
}

\author{
STEPHEN J. GARDINER
}

(Communicated by Irwin Kra)

\begin{abstract}
Let $\Omega=\mathbb{R}^{n} \backslash E$, where $E$ is a closed subset of the hyperplane $\left\{x_{n}=0\right\}$ and every point of $E$ is regular for the Dirichlet problem on $\Omega$. Further, let $\alpha_{k}$ denote the $(n-1)$-dimensional measure of the set $\{X \in$ $\left.\Omega: x_{n}=0, e^{k}<|X|<e^{k+1}\right\}$. It is known that the cone, $\mathscr{P}_{E}$, of positive harmonic functions on $\Omega$ which vanish on $E$ has dimension 1 or 2 . In this paper it is shown that if $\sum e^{-n k} \alpha_{k}^{n /(n-1)}<+\infty$, then $\operatorname{dim} \mathscr{P}_{E}=2$. This result, which in the case $n=2$ implies a recent theorem of Segawa, is also shown to be sharp.
\end{abstract}

\section{INTRODUCTION AND RESULTS}

Points of $\mathbb{R}^{n}(n \geq 2)$ are denoted by $X=\left(X^{\prime}, x_{n}\right)$, where $X^{\prime} \in \mathbb{R}^{n-1}$. We call $\Omega$ a Denjoy domain if $\Omega=\mathbb{R}^{n} \backslash E$, where $E$ is a nonempty closed proper subset of the hyperplane $\left\{x_{n}=0\right\}$ such that each point of $E$ is regular for the Dirichlet problem in $\Omega$.

Let $\mathscr{P}_{E}$ denote the cone of positive harmonic functions on $\Omega$ which vanish on $E$. It is known (see [1] or [2]) that either all functions in $\mathscr{P}_{E}$ are proportional or $\mathscr{P}_{E}$ is generated by two linearly independent minimal harmonic functions. (A positive harmonic function $u$ on $\Omega$ is called minimal if any other positive harmonic function $v$ on $\Omega$ satisfying $v \leq u$ is proportional to $u$.) We describe these cases by writing $\operatorname{dim} \mathscr{P}_{E}=1$ and $\operatorname{dim} \mathscr{P}_{E}=2$ respectively.

Roughly speaking, $\operatorname{dim} \mathscr{P}_{E}=2$ if the set $\left\{x_{n}=0\right\} \backslash E$ is "sufficiently sparse near infinity". Several results in this direction can be found in Benedicks [2]. Recently Segawa [4], working in the complex plane, added the following.

Theorem A. Let $n=2$. If there exists $\lambda>\frac{1}{2}$ such that

$$
\int_{\{x:(x, 0) \in \Omega,|x| \geq t\}} x^{-2} d x=O\left(t^{-1}[\log t]^{-\lambda}\right) \quad(t \rightarrow+\infty),
$$

then $\operatorname{dim} \mathscr{P}_{E}=2$.

(In fact, Segawa stated his result for harmonic functions with pole at the origin rather than at infinity. The above is an equivalent formulation based on inversion in the unit circle.)

Received by the editors December 1, 1988.

1980 Mathematics Subject Classification (1985 Revision). Primary 31B05, 30F25. 
The purpose of this paper is to provide a result of this type for $\mathbb{R}^{n}$, which in the case $n=2$ improves Theorem A. We will also show that our condition is sharp.

For each $k \in \mathbb{N}$ let

$$
F_{k}=\left\{X^{\prime} \in \mathbb{R}^{n-1}:\left(X^{\prime}, 0\right) \in \Omega \text { and } e^{k}<\left|X^{\prime}\right|<e^{k+1}\right\},
$$

and put $\alpha_{k}=\left|F_{k}\right|$, the $(n-1)$-dimensional Lebesgue measure of $F_{k}$. Our main result is as follows.

Theorem 1. If $\sum e^{-n k} \alpha_{k}^{n /(n-1)}<+\infty$, then $\operatorname{dim} \mathscr{P}_{E}=2$.

The proof of Theorem 1 relies on the work of Benedicks mentioned above and a balayage-type argument. Details can be found in $\S 2$. To see that Theorem 1 includes Theorem A, note that if $n=2$ and (1) hold, then

$$
e^{-k} \alpha_{k} \leq e^{2+k} \int_{F_{k}} x^{-2} d x=O\left(k^{-\lambda}\right) \quad(k \rightarrow \infty),
$$

so $\sum e^{-2 k} \alpha_{k}^{2}<+\infty$.

In the case $n \geq 3$ it is known [2, Corollary 1] that if $E$ omits an infinite $(n-1)$-dimensional circular cone, then $\operatorname{dim} \mathscr{P}_{E}=1$. The following result shows that it is sufficient for $E$ to omit a certain sequence of $(n-1)$-dimensional balls. It also establishes the sharpness of Theorem 1.

Theorem 2. Let $\left(r_{k}\right)$ be a sequence of nonnegative real numbers such that $\sum e^{-n k} r_{k}^{n}=+\infty$. If

$$
E=\left\{x_{n}=0\right\} \backslash \bigcup_{k=1}^{\infty}\left\{\left(X^{\prime}, 0\right):\left|X^{\prime}-\left(2 e^{k}, 0, \ldots, 0\right)\right|<r_{k}\right\},
$$

then $\operatorname{dim} \mathscr{P}_{E}=1$.

The proof of Theorem 2 is given in $\S 3$.

\section{Proof of Theorem 1}

2.1. We begin by stating a result of Benedicks on which our proofs rely. For each $X^{\prime} \in \mathbb{R}^{n-1}$ let $K\left(X^{\prime}\right)$ be the open cube in $\mathbb{R}^{n}$ with center $\left(X^{\prime}, 0\right)$ and side $e^{-1}\left|X^{\prime}\right|$, all sides being parallel to the coordinate hyperplanes. Further, let $\Omega\left(X^{\prime}\right)=K\left(X^{\prime}\right) \backslash E$ and $\beta_{E}\left(X^{\prime}\right)$ denote the harmonic measure of $\partial K\left(X^{\prime}\right)$ on $\Omega\left(X^{\prime}\right)$ evaluated at the point $\left(X^{\prime}, 0\right)$. The result below is drawn from [2, Theorems 3 and 4].

Theorem B. The following are equivalent:

(i) $\operatorname{dim} \mathscr{P}_{E}=2$;

(ii) there exists a function $u \in \mathscr{P}_{E}$ such that $u(X) \geq\left|x_{n}\right|$ on $\mathbb{R}^{n}$;

$$
\text { (iii) } \int_{\left\{\left|X^{\prime}\right| \geq 1\right\}}\left|X^{\prime}\right|^{1-n} \beta_{E}\left(X^{\prime}\right) d X^{\prime}<+\infty \text {. }
$$


2.2. We now collect together some definitions required in the proof of Theorem 1.

Let $h$ denote the function given by $h(X)=\left|x_{n}\right|$, and let $\sigma_{n}$ be the surface area of the unit sphere in $\mathbb{R}^{n}$. Also, let

$$
F_{0}=\left\{X^{\prime} \in \mathbb{R}^{n-1}:\left(X^{\prime}, 0\right) \in \Omega \text { and }\left|X^{\prime}\right|<e\right\}
$$

and $\alpha_{0}=\left|F_{0}\right|$. For each $k \in \mathbb{N}$, we let

$$
\gamma_{k}=\sum_{j=1}^{4} \alpha_{4 k-j}
$$

and note that the hypothesis of Theorem 1 implies that the series $\sum e^{-4 n k} \gamma_{k}^{n /(n-1)}$ converges. We define the open sets

$$
G_{k}=\left[\bigcup_{j=1}^{4} F_{4 k-j}\right] \times\left(-\gamma_{k}^{1 /(n-1)}, \gamma_{k}^{1 /(n-1)}\right) \text { and } U_{k}=\bigcup_{j=1}^{k} G_{j} .
$$

(If $\gamma_{k}=0$, then $G_{k}$ is empty.)

If $f$ is a function defined on the boundary $\partial W$ of a bounded open set $W$, we use $H[W, f]$ to denote the Perron-Wiener-Brelot solution (if it exists) to the corresponding Dirichlet problem on $W$. (An account of the properties of $H[W, f]$ can be found in [3, Chapter 8].) If $f$ is defined on the hyperplane $\left\{x_{n}=0\right\}$, we similarly write $I[f]$ for the corresponding half-space Poisson integral; that is,

$$
I[f](X)=2 x_{n} \sigma_{n}^{-1} \int_{\mathbb{R}^{n-1}} \frac{f\left(Y^{\prime}, 0\right) d Y^{\prime}}{\left\{\left|X^{\prime}-Y^{\prime}\right|^{2}+x_{n}^{2}\right\}^{n / 2}} \quad\left(x_{n}>0\right) .
$$

Now suppose that $s$ is a nonnegative subharmonic function on $\mathbb{R}^{n}$. We define

$$
\left(J_{0} s\right)(X)= \begin{cases}h(X)+I[s]\left(X^{\prime},\left|x_{n}\right|\right) & \left(x_{n} \neq 0\right) \\ s(X) & \left(x_{n}=0\right)\end{cases}
$$

and

$$
\left(J_{k} s\right)(X)= \begin{cases}H\left[U_{k}, s\right](X) & \left(X \in U_{k}\right) \\ s(X) & \left(X \in \mathbb{R}^{n} \backslash U_{k}\right)\end{cases}
$$

for any $k \in \mathbb{N}$.

2.3. For any set $A \subseteq \mathbb{R}^{n-1}$, let $\chi_{A}$ denote the function valued 1 on $A \times\{0\}$ and 0 elsewhere on $\left\{x_{n}=0\right\}$. Also, let $r_{n-1}$ be the radius of the ball $B$ centered at the origin $O^{\prime}$ of $\mathbb{R}^{n-1}$ for which $|B|=1$. We will need the following simple lemma.

Lemma 1. There is a positive constant $c_{n}<1$, depending only on $n$, such that if $A$ is a measurable subset of $\mathbb{R}^{n-1}$ satisfying $|A|=1$, then

$$
I\left[\chi_{A}\right]\left(X^{\prime}, 1\right) \leq c_{n} \quad\left(X^{\prime} \in \mathbb{R}^{n-1}\right) .
$$


In fact,

$$
\begin{aligned}
I\left[\chi_{A}\right]\left(X^{\prime}, 1\right)= & \frac{2}{\sigma_{n}} \int_{A} \frac{d Y^{\prime}}{\left\{\left|X^{\prime}-Y^{\prime}\right|^{2}+1\right\}^{n / 2}} \\
\leq & \frac{2}{\sigma_{n}} \int_{\left\{Y^{\prime} \in A:\left|X^{\prime}-Y^{\prime}\right| \leq r_{n-1}\right\}} \frac{d Y^{\prime}}{\left\{\left|X^{\prime}-Y^{\prime}\right|^{2}+1\right\}^{n / 2}} \\
& +\frac{2}{\sigma_{n}}\left(r_{n-1}^{2}+1\right)^{-n / 2}\left|\left\{Y^{\prime} \in A:\left|X^{\prime}-Y^{\prime}\right|>r_{n-1}\right\}\right| \\
\leq & \frac{2}{\sigma_{n}} \int_{\left\{Y^{\prime}:\left|X^{\prime}-Y^{\prime}\right| \leq r_{n-1}\right\}} \frac{d Y^{\prime}}{\left\{\left|X^{\prime}-Y^{\prime}\right|^{2}+1\right\}^{n / 2}} \\
= & I\left[\chi_{B}\right]\left(O^{\prime}, 1\right) .
\end{aligned}
$$

2.4. We claim that it is enough to prc re Theorem 1 in the special case where $\alpha_{4 k}=0$ for all $k=0,1,2, \ldots$. To see this, let

$$
\begin{gathered}
D=\bigcup_{k=1}^{\infty}\left\{\left(X^{\prime}, 0\right): e^{4 k} \leq\left|X^{\prime}\right| \leq e^{4 k+1}\right\}, \\
E_{j}=D \cup\left\{\left(e^{j} X^{\prime}, 0\right):\left(X^{\prime}, 0\right) \in E\right\} \quad(j=0,1,2,3),
\end{gathered}
$$

and observe that

$$
\beta_{E_{j}}\left(X^{\prime}\right)=\beta_{E}\left(e^{-j} X^{\prime}\right) \quad\left(e^{4 k+2}<\left|X^{\prime}\right|<e^{4 k+3} ; k=0,1,2, \ldots\right) .
$$

Now assume that the special case of the theorem has been established. Then we know that $\operatorname{dim} \mathscr{P}_{E_{j}}=2$ for $j=0,1,2,3$; and so by Theorem $\mathrm{B},(2)$ holds, with $E$ replaced by any $E_{j}$. From (3) it follows that

$$
\int_{\bigcup_{k}\left\{X^{\prime}: e^{4 k+j}<\left|X^{\prime}\right|<e^{4 k+j+1}\right\}}\left|X^{\prime}\right|^{1-n} \beta_{E}\left(X^{\prime}\right) d X^{\prime}<+\infty
$$

for each $j$. Hence (2) holds, and by a further application of Theorem B, $\operatorname{dim} \mathscr{P}_{E}=2$.

In the proof of Theorem 1 we can also assume that the sum $T$ of the series $\sum e^{-4 n k} \gamma_{k}^{n /(n-1)}$ satisfies

$$
c_{n}+2 T \sigma_{n}^{-1}\left(e^{-3}-e^{-7 / 2}\right)^{-n}<1,
$$

for the convergence of (2) is unaffected when we replace $E$ by $E \cup\left\{\left(X^{\prime}, 0\right):\left|X^{\prime}\right|\right.$ $\leq a\}$ for any $a>0$. The left-hand side of (4) will be denoted by $d_{n}$.

2.5. In the light of $\S 2.4$ it remains to prove Theorem 1 under the additional assumptions that (4) holds and that $\alpha_{4 k}=0$ for all $k=0,1,2, \ldots$.

Let $k \in \mathbb{N}$ and suppose that $s$ is a nonnegative subharmonic function on $\mathbb{R}^{n}$ which vanishes on $E \cup\left\{\left(X^{\prime}, 0\right):\left|X^{\prime}\right| \geq e^{4 k-4}\right\}$. Suppose also that the $\delta$-subharmonic function $s-h$ is bounded above on $\mathbb{R}^{n}$. Then the function $S=J_{k} s$ is also nonnegative and subharmonic on $\mathbb{R}^{n}$ (the regularity of $U_{k}$ for the Dirichlet problem follows from the regularity of $\Omega$ ); we have $S \geq s$; the 
function $S-h$ is bounded above; and $S$ vanishes on $E \cup\left\{\left(X^{\prime}, 0\right):\left|X^{\prime}\right| \geq\right.$ $\left.e^{4 k}\right\}$. A Phragmén-Lindelöf argument applied to each half-space now shows that $J_{0} S \geq S$ on $\left\{X: x_{n} \neq 0\right\}$ and thus that $J_{0} S$ is subharmonic on $\mathbb{R}^{n}$. Again $J_{0} S-h$ is bounded above and $J_{0} S$ vanishes on $E \cup\left\{\left(X^{\prime}, 0\right):\left|X^{\prime}\right| \geq e^{4 k}\right\}$.

It follows that the functions $\left(s_{k}\right)$ defined inductively by

$$
s_{0}=h ; \quad s_{2 k-1}=J_{k} s_{2 k-2} ; \quad s_{2 k}=J_{0} s_{2 k-1}
$$

are all nonnegative and subharmonic on $\mathbb{R}^{n}$, vanish on $E$, and form an increasing sequence.

2.6. To prove that $\lim _{k \rightarrow \infty} s_{k}$ is finite, we establish the following lemma.

Lemma 2. Let $k \in \mathbb{N}$. If there is a positive constant $C$ such that

$$
s_{2 k-1}\left(X^{\prime}, 0\right) \leq C \gamma_{j}^{1 /(n-1)} \quad\left(\left(X^{\prime}, 0\right) \in G_{j} ; j \in \mathbb{N}\right),
$$

then

$$
s_{2 k+1}\left(X^{\prime}, 0\right) \leq\left(1+C d_{n}\right) \gamma_{j}^{1 /(n-1)} \quad\left(\left(X^{\prime}, 0\right) \in G_{j} ; j \in \mathbb{N}\right) .
$$

To see this, suppose (5) holds, let $j \in \mathbb{N}$ be such that $\gamma_{j} \neq 0$, and let

$$
K=\left\{X: e^{(8 j-7) / 2}<\left|X^{\prime}\right|<e^{(8 j+1) / 2} \text { and }\left|x_{n}\right|<\gamma_{j}^{1 /(n-1)}\right\} .
$$

Using the fact that $\alpha_{4 j-4}=0=\alpha_{4 j}$, we have, for any $X \in \partial K$,

$$
\begin{gathered}
2 x_{n} \sigma_{n}^{-1} \int_{\left\{Y^{\prime} \in \mathbb{R}^{n-1}:\left(Y^{\prime}, 0\right) \notin K\right\}} \frac{s_{2 k-1}\left(Y^{\prime}, 0\right) d Y^{\prime}}{\left\{\left|X^{\prime}-Y^{\prime}\right|^{2}+x_{n}^{2}\right\}^{n / 2}} \\
\leq 2 \sigma_{n}^{-1} \gamma_{j}^{1 /(n-1)} C\left\{\left[e^{(8 j-7) / 2}-e^{4 j-4}\right]^{-n} \sum_{i=1}^{j-1} \gamma_{i}^{n /(n-1)}\right. \\
\left.+\sum_{i=j+1}^{k}\left[e^{4 i-3}-e^{(8 j+1) / 2}\right]^{-n} \gamma_{i}^{n /(n-1)}\right\} \\
\leq 2 \sigma_{n}^{-1} \gamma_{j}^{1 /(n-1)} C\left\{\left[e^{1 / 2}-1\right]^{-n} \sum_{i=1}^{j-1} e^{-4 n i} \gamma_{i}^{n /(n-1)}\right. \\
\left.\quad+\left[e^{-3}-e^{-7 / 2}\right]^{-n} \sum_{i=j+1}^{k} e^{-4 n i} \gamma_{i}^{n /(n-1)}\right\} \\
\leq 2 \sigma_{n}^{-1} \gamma_{j}^{1 /(n-1)} C\left[e^{-3}-e^{-7 / 2}\right]^{-n} T,
\end{gathered}
$$

where $T$ is as defined in $\S 2.4$. Also, using Lemma 1 and the dilation $X \mapsto$ $\gamma_{j}^{1 /(n-1)} X$, we have

$$
\begin{aligned}
2 x_{n} \sigma_{n}^{-1} & \int_{\left\{Y^{\prime} \in \mathbb{R}^{n-1}:\left(Y^{\prime}, 0\right) \in K\right\}} \frac{s_{2 k-1}\left(Y^{\prime}, 0\right) d Y^{\prime}}{\left\{\left|X^{\prime}-Y^{\prime}\right|^{2}+x_{n}^{2}\right\}^{n / 2}} \\
& \leq \gamma_{j}^{1 /(n-1)} C \max \left\{c_{n}, 2 \sigma_{n}^{-1}\left[e^{-3}-e^{-7 / 2}\right]^{-n} e^{-4 n j} \gamma_{j}^{n /(n-1)}\right\} \\
& =\gamma_{j}^{1 /(n-1)} C c_{n} \quad(X \in \partial K) .
\end{aligned}
$$


(The last step is possible since, by the final paragraph of $\S 2.4$, we can assume $j$ to be sufficiently large to achieve this.) Hence, if $X \in \partial K$,

$$
\begin{aligned}
s_{2 k}(X) & =\left(J_{0} s_{2 k-1}\right)(X) \\
& \leq \gamma_{j}^{1 /(n-1)}\left\{1+C\left[2 T \sigma_{n}^{-1}\left(e^{-3}-e^{-7 / 2}\right)^{-n}+c_{n}\right]\right\} \\
& =\gamma_{j}^{1 /(n-1)}\left(1+C d_{n}\right),
\end{aligned}
$$

where $d_{n}$ is as defined in $\S 2.4$. It follows that, if $\left(X^{\prime}, 0\right) \in G_{j}$, then

$$
\begin{aligned}
s_{2 k+1}\left(X^{\prime}, 0\right) & =\left(J_{k+1} s_{2 k}\right)\left(X^{\prime}, 0\right) \\
& = \begin{cases}H\left[G_{j}, s_{2 k}\right]\left(X^{\prime}, 0\right) & (j \leq k+1) \\
0 & (j>k+1)\end{cases} \\
& \leq \max \left\{s_{2 k}(Y): Y \in \partial K\right\} \\
& \leq \gamma_{j}^{1 /(n-1)}\left(1+C d_{n}\right),
\end{aligned}
$$

the penultimate step being a consequence of the maximum principle and the fact that $G_{j} \subseteq K$. The lemma is now established.

2.7. We now complete the proof of Theorem 1. Since

$$
s_{1}\left(X^{\prime}, 0\right)=\left(J_{1} h\right)\left(X^{\prime}, 0\right) \begin{cases}\leq \gamma_{1}^{1 /(n-1)} & \left(\left(X^{\prime}, 0\right) \in G_{1}\right) \\ =0 & \left(\left(X^{\prime}, 0\right) \notin G_{1}\right)\end{cases}
$$

it follows from Lemma 2 that

$$
s_{2 k+1}\left(X^{\prime}, 0\right) \leq\left(1+d_{n}+d_{n}^{2}+\cdots+d_{n}^{k}\right) \gamma_{j}^{1 /(n-1)} \quad\left(\left(X^{\prime}, 0\right) \in G_{j} ; j \in \mathbb{N}\right) .
$$

Since $d_{n}<1$, it follows that the function $s=\lim _{k \rightarrow \infty} s_{k}$ is locally bounded on $\left\{x_{n}=0\right\}$. Further, $s_{2 k-1}$ is harmonic in $U_{k}$, so $s=\lim s_{2 k-1}$ is harmonic in $\bigcup_{j} G_{j}$. We can assume this latter set to be nonempty (otherwise the theorem is vacuously true), so there are points of the half-spaces $\left\{x_{n}>0\right\}$ and $\left\{x_{n}<0\right\}$ where $s$ is finite. Since $s_{2 k}$ is harmonic in $\left\{x_{n} \neq 0\right\}$ for each $k$, so is $s=$ $\lim s_{2 k}$. In fact, applying the monotone convergence theorem to the equation $s_{2 k}=J_{0} s_{2 k-1}$, we find that

$$
s(X)=h(X)+I[s]\left(X^{\prime},\left|x_{n}\right|\right) \quad\left(x_{n} \neq 0\right),
$$

and so $s$ is locally bounded on $\mathbb{R}^{n}$.

We have now established that $s$ is harmonic on the set $\mathbb{R}^{n} \backslash(E \cup L)$, where

$$
L=\left\{\left(X^{\prime}, 0\right): \log \left|X^{\prime}\right| \in \mathbb{N}\right\} .
$$

Since $L$ is polar and $s$ is locally bounded on $\mathbb{R}^{n}$, it follows that $s$ can be redefined (if necessary) on $L \cap E$ in such a way that $s$ is harmonic on $\mathbb{R}^{n} \backslash E$.

Now let $a>0$ and $B=\{X \in \Omega:|X|<a\}$. Since each $s_{k}$ is subharmonic on $\mathbb{R}^{n}$ we have $s_{k} \leq H\left[B, s_{k}\right]$ on $B$, whence $s \leq H[B, s]$ on $B$ by monotone convergence. Since, further, each $s_{k}$ vanishes on $E$, it follows that $s$ continuously vanishes on the set $\{X \in E:|X|<a\}$. (Any point of the latter set is 
regular for the Dirichlet problem on $B$.) Thus, as $a$ can be arbitrarily large, $s$ continuously vanishes on $E$. Also, $s \geq s_{0}=h$. The equivalence of (i) and (ii) in Theorem B now shows that $\operatorname{dim} \mathscr{P}_{E}=2$.

\section{Proof of TheOREM 2}

Let $E^{\prime}=\left\{\left(X^{\prime}, 0\right):\left|X^{\prime}\right| \geq 1\right\}$ and let $u \in \mathscr{P}_{E^{\prime}}$ be symmetric in $x_{n}$. Clearly there is a positive constant $c$ such that

$$
u(X)=c\left|x_{n}\right|+I[u]\left(X^{\prime},\left|x_{n}\right|\right) \quad\left(x_{n} \neq 0\right)
$$

and so, multiplying by a suitable factor, we can assume that $u(X) \leq 1+\left|x_{n}\right|$ for all $X \in \mathbb{R}^{n}$.

Let $\left(r_{k}\right)$ and $E$ be as in the statement of Theorem 2 and let $2 \rho_{k}=$ $\min \left\{r_{k}, e^{k}\right\}$. Clearly $\sum e^{-n k} \rho_{k}^{n}=+\infty$. Let

$$
B_{k}=\left\{X^{\prime} \in \mathbb{R}^{n-1}:\left|X^{\prime}-\left(2 e^{k}, 0, \ldots, 0\right)\right|<\rho_{k}\right\} .
$$

If $X^{\prime} \in B_{k}$, then $\beta_{E}\left(X^{\prime}\right) \geq \beta_{\star}\left(X^{\prime}\right)$, where $\beta_{\star}\left(X^{\prime}\right)$ is the harmonic measure of $\partial K\left(X^{\prime}\right)$ on the set

$$
W=K\left(X^{\prime}\right) \backslash\left\{\left(Y^{\prime}, 0\right):\left|Y^{\prime}-X^{\prime}\right| \geq \rho_{k}\right\}
$$

evaluated at the point $\left(X^{\prime}, 0\right)$. Applying the maximum principle to $W$, it follows that

$$
\beta_{\star}\left(X^{\prime}\right) \geq\left(1+\frac{\left|X^{\prime}\right|}{2 e \rho_{k}}\right)^{-1} u(0),
$$

whenever $\rho_{k} \neq 0$. Hence

$$
\begin{aligned}
\int_{\left\{\left|X^{\prime}\right| \geq 1\right\}}\left|X^{\prime}\right|^{1-n} \beta_{E}\left(X^{\prime}\right) d X^{\prime} & \geq u(0) \sum_{\rho_{k} \neq 0} \int_{B_{k}}\left|X^{\prime}\right|^{1-n}\left(1+\frac{\left|X^{\prime}\right|}{2 e \rho_{k}}\right)^{-1} d X^{\prime} \\
& \geq \frac{\sigma_{n-1}}{(n-1)} u(0) \sum_{\rho_{k} \neq 0} e^{(k+1)(1-n)} \rho_{k}^{n-1}\left(1+\frac{e^{k}}{2 \rho_{k}}\right)^{-1} \\
& \geq \frac{\sigma_{n-1}}{(n-1)} u(0) e^{1-n} \sum_{k=1}^{\infty} e^{-n k} \rho_{k}^{n} \\
& =+\infty .
\end{aligned}
$$

It now follows from Theorem B that $\operatorname{dim} \mathscr{P}_{E}=1$.

\section{REFERENCES}

1. A. Ancona, L'ne propriété de la compactification de Martin d'un domaine euclidien, Ann. Inst. Fourier (Grenoble) 29 (1979), 71-90.

2. M. Benedicks, Positive harmonic functions vanishing on the boundary of certain domains in $\mathbf{R}^{n}$, Ark. Mat. 18 (1980), 53-71. 
3. L. L. Helms, Introduction to potential theory, Krieger, New York, 1975.

4. S. Segawa, Martin boundaries of Denjoy domains, Proc. Amer. Math. Soc. 103 (1988), 177183.

Department of Mathematics, University College, Dublin 4, Ireland 